\title{
Evolutionary Planner of 3D Trajectories
}

\section{Petar Ćurković \& Lovro Čehulić}
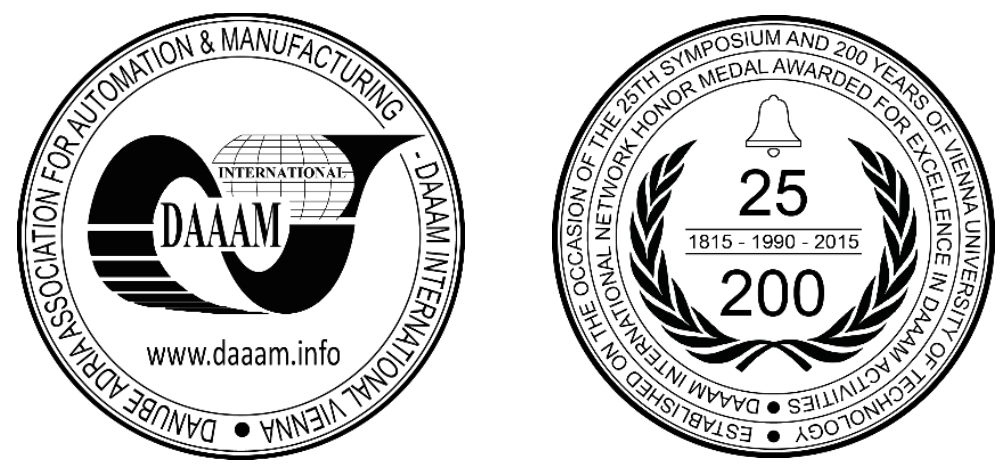

This Publication has to be referred as: Curkovic, P[etar] \& Cehulic, L[ovro] (2018). Evolutionary Planner of 3D Trajectories, Proceedings of the 29th DAAAM International Symposium, pp.0400-0405, B. Katalinic (Ed.), Published by DAAAM International, ISBN 978-3-902734-20-4, ISSN 1726-9679, Vienna, Austria DOI: $10.2507 / 29$ th.daaam.proceedings.058

\begin{abstract}
This paper presents a method based on evolutionary algorithms for the planning of robotic trajectories both in $2 \mathrm{D}$ and 3D space. Space can be arbitrary shaped containing randomly sized and distributed obstacles. Different methods were implemented and evaluated for improving the speed and the level of the convergence of the algorithm. It is shown that evolutionary algorithm in its simplest form cannot effectively solve this problem in 3D. A thorough statistical analysis is performed for various instances of the algorithm, and the best algorithmic procedures are identified, documented, and explained in the paper.
\end{abstract}

Keywords: motion planning; evolutionary algorithms; artificial intelligence

\section{Introduction}

At present, we are trying to optimise everything, from processing and manufacturing down to our daily lives. Over the years, many optimisation techniques were created, with evolutionary algorithms being significantly researched and used area of optimisation. Evolutionary algorithms are powerful optimisation tool based on genetical evolution, and they are becoming increasingly popular because of their applicability and adaptability.

In this paper, an evolutionary algorithm is used to test how different diversity maintenance methods affect speed and the level of convergence of the algorithm. In order to do that, the algorithm was used to find the solution of a difficult real-world problem [1],[2],[3],[4]: find a feasible and optimized path inside of 2D and 3D space filled with obstacles. The pathway must end in the designated area, and it is not allowed to have collisions with obstacles. Different methods were developed, used and compared, and the results are presented later in the paper. This setup presented in the paper can be directly applied to industrial robot - allowing the robot to follow the trajectory found by the algorithm, guiding mobile robots through arbitrary labyrinths, and movement of drones in space.

\section{Evolutionary algorithm and testing environment}

To test how the diversity maintenance methods influence the performance of the algorithm, three instances were made and compared. First one is the basic algorithm while other two use fitness sharing and crowding, respectively, to control the diversity of the population. 
Every element of the algorithms, other than parent selection, is identical to make their results comparable. Furthermore, they are tested on the same labyrinths with the same obstacle placements and starting and goal positions. The population is a matrix (1), (2), where $m$ represents the number of individuals and $n$ represents the length of the genome. Population size $m$ is set to 50 members in each algorithm, while genome length $n$ is set to 10 which is the required number of steps from the start to the end of the labyrinth with no collisions. For the 2D labyrinth, the genome is made of randomly generated numbers ranging from 1 to 4 (1). Each number represents different movement through the labyrinth, where 1 and 2 describe movement in the positive and negative direction of the $\mathrm{x}$-axis, respectively, while 3 and 4 describe movement in the positive and negative direction of the $y$-axis, respectively.

$$
\text { population }=\left[\begin{array}{cccc}
\operatorname{rand}(4)_{11} & \operatorname{rand}(4)_{12} & \cdots & \operatorname{rand}(4)_{1 n} \\
\operatorname{rand}(4)_{21} & \operatorname{rand}(4)_{22} & \cdots & \operatorname{rand}(4)_{2 n} \\
\vdots & \vdots & \ddots & \vdots \\
\operatorname{rand}(4)_{m 1} & \operatorname{rand}(4)_{m 1} & \cdots & \operatorname{rand}(4)_{m n}
\end{array}\right]
$$

For the 3D labyrinth, the genome has additional numbers 5 and 6 (2), they describe movement in the positive and negative direction of the z-axis, respectively.

$$
\text { population }=\left[\begin{array}{cccc}
\operatorname{rand}(6)_{11} & \operatorname{rand}(6)_{12} & \cdots & \operatorname{rand}(6)_{1 n} \\
\operatorname{rand}(6)_{21} & \operatorname{rand}(6)_{22} & \cdots & \operatorname{rand}(6)_{2 n} \\
\vdots & \vdots & \ddots & \vdots \\
\operatorname{rand}(6)_{m 1} & \operatorname{rand}(6)_{m 1} & \cdots & \operatorname{rand}(6)_{m n}
\end{array}\right]
$$

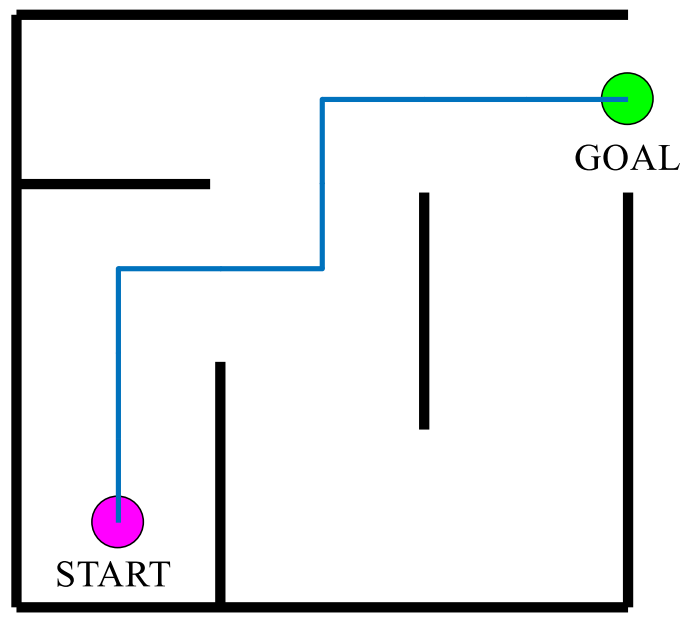

a)

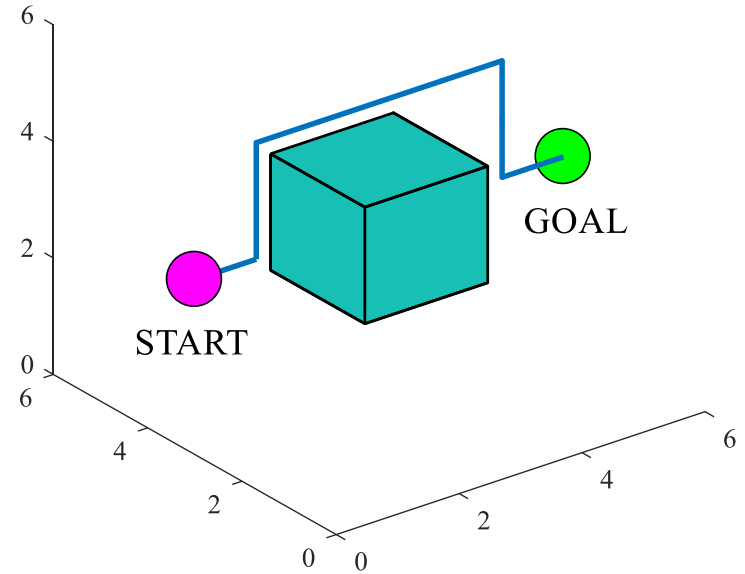

b)

Fig. 1. Example of a discovered path: a) 2D space, b) 3D space

Figure 1 represents only an example of many possible paths that do not have collisions in both 2D and 3D space. In the starting phase of the algorithm, many members of the population do not reach the goal, and later when they do, it is not without a collision. Therefore, every member of the population $i$ is evaluated and rated using a fitness value $F$ according to their performance. Fitness value depends on the distance from the end of the labyrinth and the number of collisions with the obstacles, also small penalty $r p$ is given if individual returns to the previous position.

$$
\begin{aligned}
& F(i)=f 1+f 2-r p \\
& f 1=\frac{60+\text { distance }}{1+\text { distance }}
\end{aligned}
$$

Equation (4) represents a reward for the distance from the end of the labyrinth, smaller the distance, greater the reward to the maximum of 60 if the distance is 0 . 


$$
f 2=40-\frac{40 \cdot(\text { number of collisions })}{n}+\left\{\frac{40}{n} \cdot \frac{\text { first step } \text { with collision }}{n} \quad \begin{array}{cl}
\text { if there is no collision } \\
\text { if there is collision }
\end{array}\right.
$$

Equation (5) represents how the collisions with obstacles are rewarded, individuals that strike fewer obstacles get a bigger reward, and what's more, those individuals that strike an obstacle in later step also receive a better reward. The maximum value of $f 2$ is 40 , what brings maximum fitness value of individuals to 100 . Return penalty (6) was added because it was noticed that it slightly speeds up the algorithm.

$$
r p= \begin{cases}0, & \text { if there are no returns } \\ 10, & \text { if there are returns }\end{cases}
$$

Regarding the rest of the algorithm, it uses standard one-point crossover recombination operator and random resetting mutation operator. Parent selection is done using fitness proportional selection with the roulette wheel rule, while survivor selection is age-based with the use of elitism. Every algorithm is limited to 500 generations. If the goal is not reached in 500 generations, the algorithm is stopped, and it is considered that it failed even though it could potentially reach the goal in a later generation.

\section{Diversity maintenance}

The idea is to force the population to maintain different when doing selection or replacement. There are two most commonly used methods, fitness sharing [5], where fitness value of each individual is adjusted before the selection to separate them in niches in proportion to the niche fitness, and crowding [6], where individuals are distributed uniformly amongst niches using distance-based selection. It should be noted that both methods use global parent selection and there is nothing that prevents recombination of parents from different niches. Both methods belong to the general approach of diversity maintenance that is of utmost importance for evolutionary inspired algorithms to work successfully dealing with hard optimization tasks [7].

\subsection{Fitness sharing}

This method is based on the idea that "sharing" of individual fitness values before the selection controls the number of individuals in each niche. In this method, every possible pairing of individuals $i$ and $j$ is considered, and then a distance was calculated between them. The fitness $F$ of each individual $i$ is then adapted according to the number of individuals falling within some pre-specified distance $\sigma_{\text {share }}$ using a power-law distribution:

$$
\begin{aligned}
& F^{\prime}(i)=\frac{F(i)}{\sum_{j} \operatorname{sh}(d(i, j))} \\
& \operatorname{sh}(d)=\left\{\begin{array}{cl}
1-\left(d / \sigma_{\text {share }}\right)^{\alpha} & \text { if } d \leq \sigma_{\text {share }}, \\
0 & \text { otherwise }
\end{array}\right.
\end{aligned}
$$

The sharing function $\operatorname{sh}(d)$ is a function of the distance. Constant value $\alpha$ determines the shape of the sharing function and it was taken as $\alpha=1$ to make the function linear. The last parameter was share radius $\sigma_{\text {share }}$ which defines how many niches can be maintained. It is recommended, [8], to use its value in the range of 5-10, in this algorithm value of 10 was taken to have larger diversity. Even though it is preferred to use phenotypic distance value $d$, trial-anderror proved that using a difference between fitness values of individuals as their distance gives better results and faster convergence of the algorithm.

With the use of fitness sharing, members with a lower fitness value have the probability of becoming parents higher than it is the case in the basic algorithm. This way we have a larger gene pool [9] which directly increases the diversity of the population.

\subsection{Crowding}

This method relies on the fact that offspring are likely to be similar to their parents. In this algorithm, parent population is evaluated and then randomly paired. Each pair produces two offspring by recombination, after which offspring are mutated and then evaluated. If parents are denoted as $p$, and offspring as $o$, distances: $d\left(p_{1}, o_{1}\right), d\left(p_{1}, o_{2}\right)$, $d\left(p_{2}, o_{1}\right)$ and $d\left(p_{2}, o_{2}\right)$ between parents and offspring need to be calculated. After that competition pairs are identified.

$$
\left[d\left(p_{1}, o_{1}\right)+d\left(p_{2}, o_{2}\right)\right] \leq\left[d\left(p_{1}, o_{2}\right)+d\left(p_{2}, o_{1}\right)\right]
$$


If (9) is true, the competition is between $p_{1-O_{1}}$ and $p_{2-O_{2}}$, otherwise, the competition is between $p_{1}-O_{2}$ and $p_{2}-O_{1}$. There is no competition between offspring, they only compete with the most similar parent. Individuals with higher fitness values stay in the population and losers are discarded.

In comparison with previous two algorithms, this one uses different parent selection model as well as different survivor selection model. With crowding, parent population is paired in such way that every member becomes a parent. Also, survivor selection is fitness-based instead of age-based while it still uses elitism.

\section{Results}

It should be stated that algorithms, simulations and results were all created in MATLAB environment, version R2017a. In order to compare different versions of the algorithm, each algorithm was simulated 1000 times and histograms were used to represent the results. Each histogram represents how many times an algorithm found the solution on a specific generation or how many times it stopped because of reached generation limit.

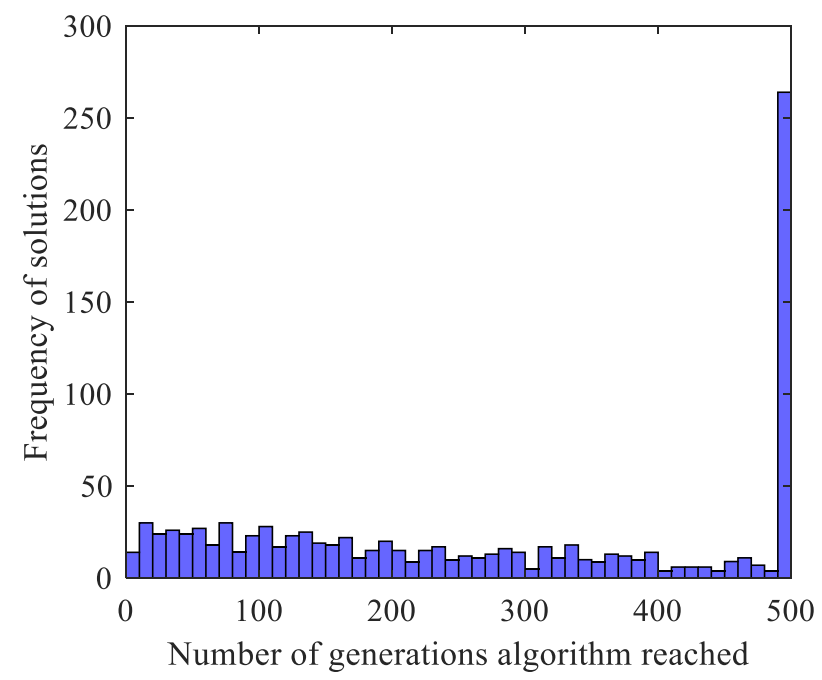

a)

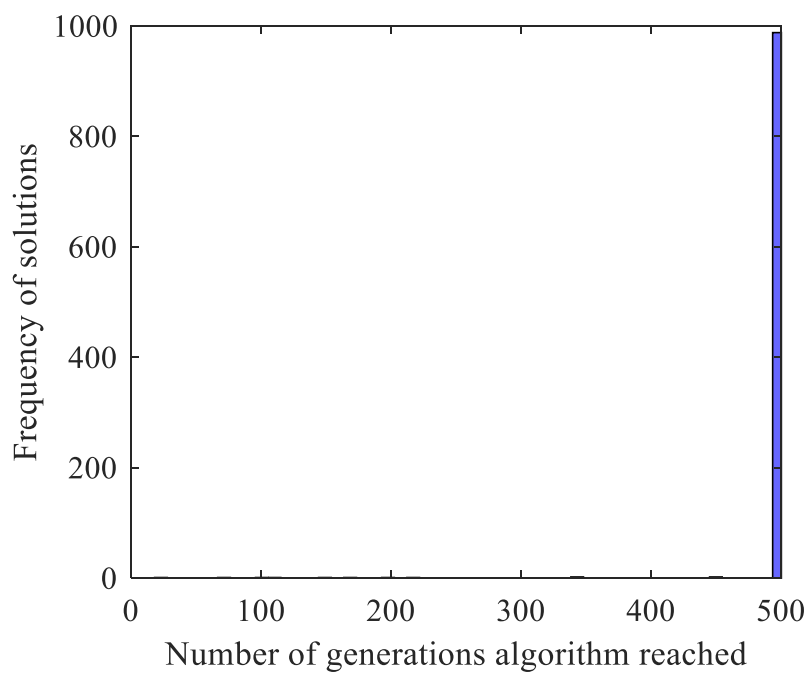

b)

Fig. 2. Basic algorithm testing results: a) 2D space, b) 3D space

Testing results of the basic algorithm (Fig. 2) show that basic algorithm is very unreliable. These results show that even though it is moderately successful in finding the wanted solution in 2D space, with the basic algorithm we can't predict how good the found solution is. Solutions are spread almost evenly in the generation span, while in $26.4 \%$ of simulations algorithm stopped because it reached the 500 generation limit. 3D problem is significantly harder to solve than the $2 \mathrm{D}$ problem, as it is clear from the results.

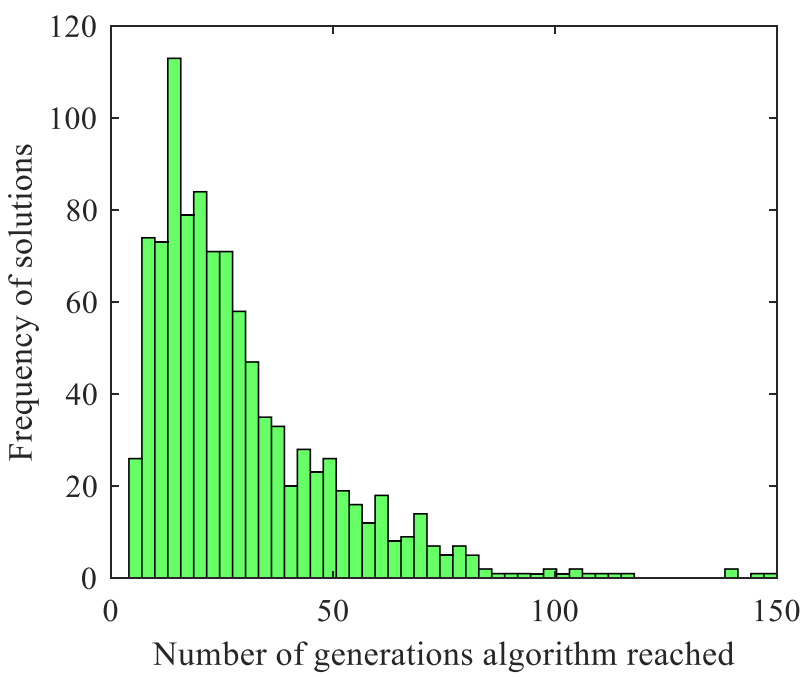

a)

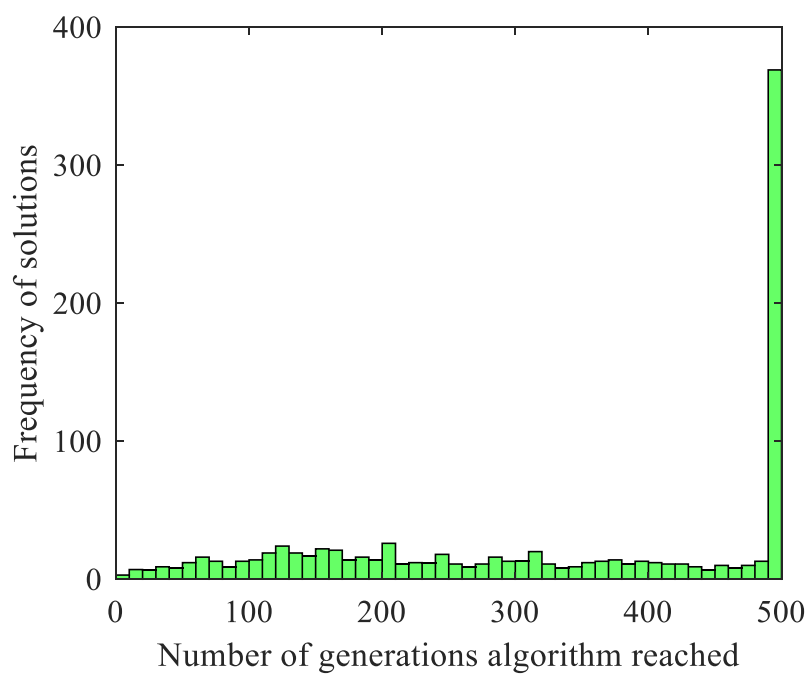

b)

Fig. 3. Fitness sharing algorithm testing results: a) 2D space, b) 3D space 
Even though the 3D problem was intentionally set up to be easiest possible, with only one obstacle, basic algorithm failed to find a path without collisions in $98.8 \%$ of simulations. When we look at the testing results of algorithms with fitness sharing (Fig. 3), it is clear how much this diversity maintenance method improves the basic algorithm. On a 2D problem, the algorithm found the solution in the defined limit of 500 generations in every simulation. What's more, this algorithm found a solution in less than 30 generations on average. Looking at the results of the 3D problem we can see a similar result as with basic algorithm on the $2 \mathrm{D}$ problem. Solutions are spread almost evenly in the entire generation span, and in many cases, the algorithm failed to find a solution.

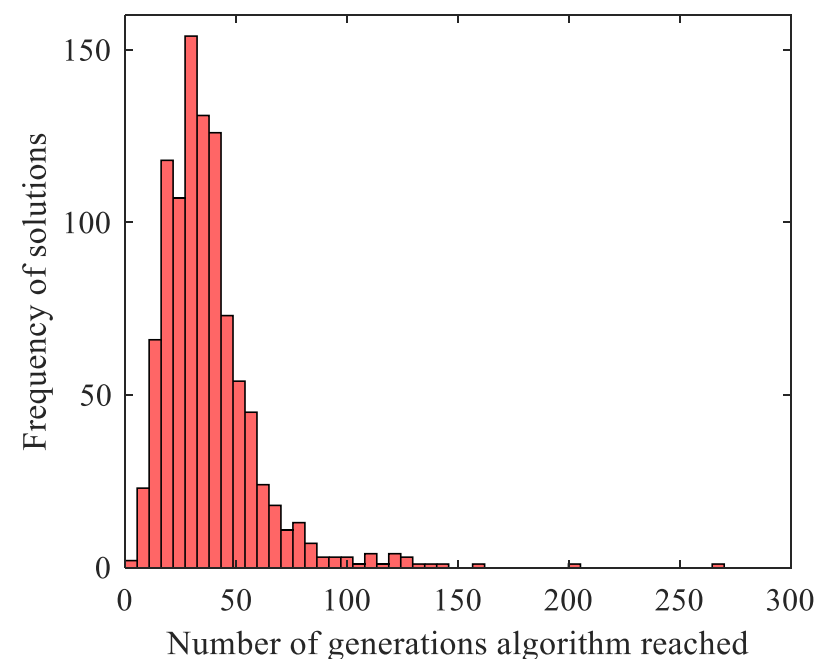

a)

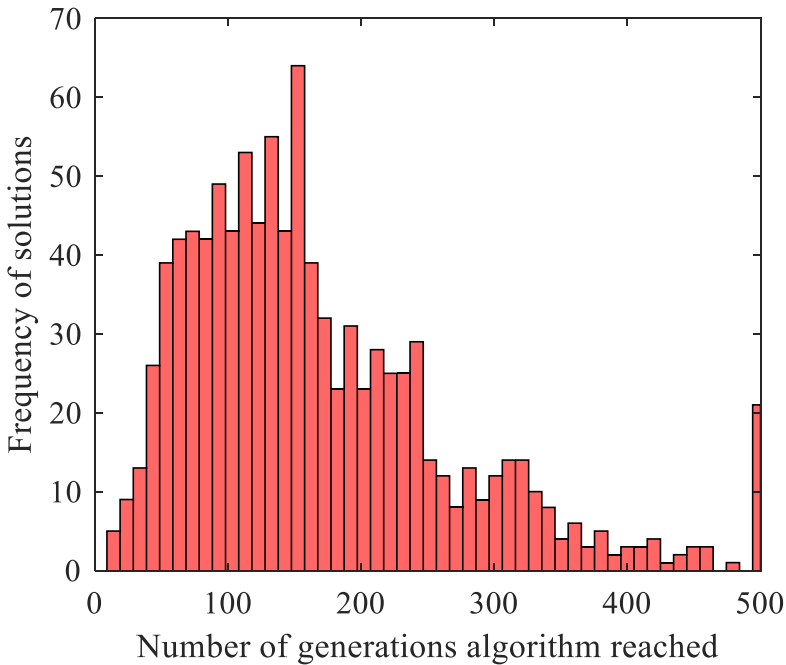

b)

Fig. 4. Crowding algorithm testing results: a) 2D space, b) 3D space

From the results of the testing of the algorithm with crowding (Fig. 4), it is clear that this the most successful of the tested algorithms. In the 2D problem, a solution was, similar as in fitness sharing algorithm, also found in every simulation, and results resemble those of the algorithm with fitness sharing. However, the results from the 3D problem show that crowding has a great advantage in comparison to fitness sharing when it comes to more challenging problems. In the 3D problem, crowding failed to find a solution in the 500 generation limit only in 21 simulations what shows the reliability of this method.

\begin{tabular}{|c|c|c|c|c|c|c|}
\hline Algorithm & $\begin{array}{c}\text { Number of } \\
\text { simulations }\end{array}$ & $\begin{array}{c}\text { Number of } \\
\text { stopped } \\
\text { simulations }\end{array}$ & $\begin{array}{c}\text { Minimal } \\
\text { reached } \\
\text { generation }\end{array}$ & $\begin{array}{c}\text { Maximal } \\
\text { reached } \\
\text { generation }\end{array}$ & $\begin{array}{c}\text { Average } \\
\text { number of } \\
\text { generations }\end{array}$ & $\begin{array}{c}\text { Standard } \\
\text { deviation }\end{array}$ \\
\hline Basic 2D & 1000 & 264 & 4 & 500 & 272.291 & 177.2984 \\
\hline Fitness sharing 2D & 1000 & 0 & 4 & 150 & 29.271 & 20.6629 \\
\hline Crowding 2D & 1000 & 0 & 4 & 266 & 37.304 & 21.8409 \\
\hline Basic 3D & 1000 & 988 & 21 & 500 & 496.609 & 34.3777 \\
\hline Fitness sharing 3D & 1000 & 369 & 5 & 500 & 334.569 & 162.8297 \\
\hline Crowding 3D & 1000 & 21 & 10 & 500 & 167.713 & 101.3886 \\
\hline
\end{tabular}

Table 1. Testing results

\section{Conclusion}

Results show that diversity maintenance methods significantly influence the performance of evolutionary algorithms. The difference is significant even on a simple 2D problem where basic version of the algorithm has a difficulty finding a solution. Looking at the testing results of the 3D problem, the basic algorithm didn't stop only in $1.2 \%$ of simulations what can be taken as a statistical error. We can safely conclude that evolutionary algorithm in its simplest form cannot effectively solve this problem in 3D.

Regarding the fitness sharing version of the algorithm, it had very good results on the 2D problem, what's more, they are little better than the results of the version with crowding. However, it only finished $63.1 \%$ of simulations during the testing of the 3D problem in comparison to the algorithm with crowding that finished $97.9 \%$ of simulations. That is to say, crowding, as diversity maintenance method, is significantly more reliable and robust than fitness sharing. 
The question that comes to mind is why crowding gave so much better results than fitness sharing? The only probable reason is that the discrepancy comes from the difference in parent selection methods. In crowding algorithm, every single member of the population is randomly paired into parent population, while fitness sharing is modified fitness proportional selection with a roulette wheel sampling. That means that with crowding, every member of the population contributes to the gene pool of the population. Even though roulette wheel sampling provides a chance of selection depending on the fitness value, it is still stochastic method where a good member of the population can lose its place in the parent population. Further research is needed to come to a conclusion about this issue.

Our future research will be focused on implementation of explained algorithms on real-life applications, concretely to control the trajectories of industrial robots.

\section{References}

[1] Yukhimets, D[mitry]; Zuev, A[lexander] \& Gubankov, A[nton] (2017). Method of Spatial Path Planning for Mobile Robot in Unknown Environment, Proceedings of the 28th DAAAM International Symposium, pp.02580267, B. Katalinic (Ed.), Published by DAAAM International, ISBN 978-3-902734-11-2, ISSN 1726-9679, Vienna, Austria

[2] Ćurković, P.; Jerbić, B.; Stipančić, T. (2009) Swarm-based Approach to Path Planning Using Honey-bees Mating Algorithm and ART Neural Network. Solid state phenomena. 147-149; 74-80

[3] Ćurković, P.; Jerbić, B. (2007). Honey-bees optimization algorithm applied to path planning problem. International Journal of Simulation Modelling. VI, 3; 154-165

[4] Ćurković, P.; Jerbić, B.; Stipančić, T. (2013). Coordination of Robots With Overlapping Workspaces Based on Motion Co-Evolution. // International journal of simulation modelling. 12, 1; 27-38

[5] Eiben, A.E.; Smith, J.E. (2007). Introduction to Evolutionary Computing. Springer Natural Computing Series.

[6] Goldberg, David E. (1989) Genetic Algorithms in search, optimization, and machine learning. Addison Wesley.

[7] Squillero, G.; Tonda, A. (2016). Promoting Diversity in Evolutionary Algorithms: an Updated Bibliography. Proceedings oft he 2016 conference on Genetic and Evolutionary Computation Conference, p.pp 934-944, Denver, CO, USA.

[8] L. Pan; C. He; Y. Tian, H. Wang; X. Zhang and Y. Jin, (2018). "A Classification Based Surrogate-Assisted Evolutionary Algorithm for Expensive Many-Objective Optimization," in IEEE Transactions on Evolutionary Computation. doi: 10.1109/TEVC.2018.2802784

[9] Hoang L.; Han La Poutré; Bosman P. A.N. (2018). Multi-objective Gene-pool Optimal Mixing Evolutionary Algorithm with the interleaved multi-start scheme. Swarm and Evolutionary Computation, Elsevier. 